\title{
On Hair Follicle Development and Wool Production Traits in Sheep: A Review
}

\author{
Wu Sun, Shike Ma ${ }^{*}$ and Yuhong Ma \\ Academy of Animal Science and Veterinary Medicine, Qinghai University, Xining, 810016, China \\ For correspondence: msk122@sina.com \\ Received 15 September 2020; Accepted 10 October 2020; Published 10 January 2021
}

\begin{abstract}
Hair follicle and skin development is a complex biological process involving many regulatory molecules. Wool trait is a complex quantitative trait controlled by multiple genes and affected by environment. In this paper, the histomorphology of hair follicle development in sheep and the molecular mechanism of hair follicle and wool traits formation were reviewed in order to provide theoretical basis for breeding and selection of sheep wool traits. (C) 2021 Friends Science Publishers
\end{abstract}

Keywords: Sheep; Hair follicle development; Wool production traits; Transcriptome; lncRNA; GWAS

\section{Introduction}

Hair is one of the important features of animal appearance. It has a great influence on body beauty, spirit and social psychology. There are some rare hairless animals in the animal kingdom. The morphogenesis of hair follicle is complicated, and several stages are involved in it (Schneider et al. 2009). The problems relevant to the key genes (single and/or genes) affecting hair follicle development, together or singly, are worthy of further exploration and analysis. It is a challenge to locate and analyze the molecular basis of sheep wool traits by traditional candidate genes. There exists the question how to improve the accuracy of the selection of wool quality traits and the scientific bases of breeding program in sheep breeding. To answer this, the urgent task is to carry out the research on the molecular basis and regulatory mechanism in the formation process of fine wool quality traits. The present review presents a number of tasks to clarify the phenomenon. Firstly, the histomorphology of skin and hair follicles is described. Secondly, the important molecular genetic progress in sheep skin, hair follicle development and wool production traits by transcriptomics and IncRNA have been systematically described. Thirdly, the genetic variation screening and other molecular means were addressed. The purpose of this review is to provide a scientific theoretical basis for carrying out further studies relevant to fine wool sheep genetics and breeding, wool production traits and wool quality.

\section{Histomorphology of skin hair follicles}

As an important accessory organ of skin, hair follicle has its own unique physiological structure (Chong et al. 2003). Though the main structure of hair follicle is same, it varies a little among different species and genera of animals (Paus and Cotsarelis 1999). The duration of hair follicle morphogenesis varies with the animal species. Hair follicle is the skin cell which takes part in the hair growth, development and regeneration of animal fur and plays a significant role in mammals (Zhang et al. 2009). After the development of hair follicle, it will grow periodically and renew itself constantly. Therefore, it is a renewable organ. Under the control of various growth factors and molecular regulatory mechanisms, hair follicles continue to undergo a cyclical process of growth, degeneration and rest.

It was reported that sections of Inner Mongolia Albas cashmere goat embryo skin was observed and photographed under the microscope (Zhang et al. 2007). The results showed that the hair follicle structure of cashmere goat was composed of hair ball, connective tissue sheath, outer root sheath, inner root sheath and hair stem. Another study on morphological observation on the development of rat embryonic hair follicle showed that the morphogenesis of rat embryonic hair follicle occurred on the $12^{\text {th }}$ day of embryo. And was formed by the cells in the basal layer of epidermis, protruding into the dermis, and there were mesenchymal cells around it (Hao et al. 2006). Liu et al. (2015) carried out microscopic observation separately on the longitudinal and transverse sections from the skin tissues of Aohan fine wool sheep on the $90^{\text {th }}$ and $120^{\text {th }}$ day of gestational age and lambs on 1 and $30 \mathrm{~d}$ after birth, respectively. The results showed that the structure of wool sac of Aohan fine wool included connective tissue sheath, outer root sheath, inner root sheath, hair stem and hair bulb. The above-mentioned results can 
provide reference for understanding the changes in morphological structure of fine wool follicles and screening differential genes, related to wool density.

\section{Molecular genetic progress of hair follicle development and wool trait}

Research progress of candidate genes and signal pathways related to hair follicle development and wool production trait The development of hair follicle mainly includes eight stages ( Veen et al. 1999). The first and second stages of development followed the formation of basal plate and hair root, respectively. The $3-5^{\text {th }}$ stage is the formation of hair nail and the differentiation of hair follicle occurs at the 6-8th stages. Hair follicles are regulated by a variety of molecular signals in different growth cycles (Lin et al. 2006). The pathways, promoting hair follicle growth include Wnt/bcatenin, EDA/EDAR, Shh, notch and TGFB. BMP is one of the pathways that inhibit hair follicle growth. Shh is an important regulator in hair follicle development (Fuchs and Raghavan 2002). In the early embryo stage, notch affects the growth and development of hair follicles by stimulating hair follicle stem cells (Kopan and Weintraub 1993). Wnt is a germ plasm secretory protein that controls downstream target genes by interacting with receptors on cell membrane (Jamora et al. 2003). Wnt plays an irreplaceable role in the process of hair follicle formation. It participates in all aspects of hair follicle formation and plays a very important role in the development of hair follicles in almost all animals (Jamora et al. 2003). BMP family also plays an important role in the early stage of hair follicle development, and BMP4 can inhibit the development of hair basal plate (Blessing et al. 1993). Noggin and BMP4 are a pair of antagonists, which can effectively prevent the binding of BMP4 and its receptor and promote hair follicle growth (Botchkarev et al. 1999 2002). The expression of Hox gene affects the morphology of hair follicles (Packer et al. 2000; Shang et al. 2002). EGF is expressed in the outer root sheath of hair follicles and is closely related to the growth and development of keratinocytes (Nanney et al. 1984; Cros et al. 1992). EGF can delay the development and prolong the growth cycle of hair follicles (Chen et al. 2002). FGF and FGF-receptor gene expression in early hair follicle development, promote hair follicle formation. FGF5 can inhibit the growth of hair follicles and make them enter into the later growth stage (Ota et al. 2002). Related research reported that natural length and straight length of wool of modified individual owned two indel in FGF5 gene in adult Merino sheep (GM), were significantly longer than those of wild type (WT) (Hu et al. 2017). And the amount of dirty wool was also significantly higher than that of WT individuals. It indicated that FGF5 gene edited by CRISPR/cas9 can promote the growth of wool, thus increasing the length and yield of wool. In addition, the coding region of FGF5 was cloned (Liu et al. 2011) and found that FGF5 gene is highly expressed in skin tissues relative to kidney, small intestine, heart, spleen, liver, pancreas and lung. These evidences further indicate that FGF5 can be used as a candidate gene for hair length selection. TGF $\beta-2$ and TGF $\beta-1$ belong to TGF family. TGF $\beta$ - 2 can induce hair follicle development, while TGF $\beta$ 1 can inhibit hair follicle development (Bond et al. 1996; Schmidtullrich and Paus 2005). Wnt10b can induce immature epidermal cells to differentiate into hair stem and inner root sheath. Wnt10b is highly expressed in hair mother cells and inner root sheath, and promotes the continuous development of hair follicle (Mikkola and Millar 2006). In this concept, the worth mentioning is that MAP2 gene is a key gene responsible for hair follicle density and 1 missense mutation of A-to-G at rs328005415 in MAP2, causing a valine-to-methionine substitution leads to the hairless phenotype (Jiang et al. 2019). Kinesin superfamily proteins (KIFs) are a kind of molecular motors, which can combine with adenosine triphosphate (ATP) and convert the chemical energy produced by hydrolysis into mechanical energy. Related research has shown that a SNP was significantly related to the coefficient of variation of wool fiber diameter, which was located on chromosome 13 of sheep and could be identified as Kinesin family member 16B (KIF16b) gene (Wang et al. 2014). It is speculated that this gene can be used as a candidate gene for sheep wool traits. Based on the above concept, KIF16b gene family was expected to receive more and more attention in the future. At the same time, it plays a very important role in the process of intracellular material transport, which is responsible for transporting protein complexes, organelles and mRNA along microtubules to its positive electrode key proteins for basic cell activity (Hirokawa and Tanaka 2015). The abovementioned key genes that affect hair follicle development are worthy of further exploration and analysis.

\section{Progress in transcriptomics of sheep skin}

The screening of genes, regulating wool growth provides theoretical guidance for improving wool production efficiency, product quality and breeding, and also provides basis for the development of therapeutic drugs for human hair loss and other symptoms. There have been many studies on genetic polymorphism of wool and cashmere growth and regulation (Purvis and Franklin 2005; Cano et al. 2007; Bidinost et al. 2008). In mammals, several gene families, such as Wnts, TNFs, FGFs and TGFs, have been found to be involved in hair follicle development and growth (Galbraith 2010). Microarray analysis and other transcriptome studies have been successfully applied to the characterization of mouse hair follicle stem cells (Rhee et al. 2006; Janich et al. 2011). Microarray studies on different characteristics of sheep and goats, such as breast development (Bongiorni et al. 2009), goat milk quality (Ollier et al. 2007), hair follicle development (Norris et al. 2005), resistance to natural wool rot (Smith et al. 2010), and pigmentation of skin and wool (Penagaricano et al. 2012) 
have been reported. The gene expression patterns of Aohan fine wool sheep's lateral skin and groin skin were compared using gene expression microarray technology and proteomics technology (Liu et al. 2014). Results obtained showed 1494 differentially expressed probes, including 602 probes with high expression and 892 probes with low expression. Cluster analysis and gene annotation showed that the differentially expressed genes mainly bind to receptors related to multicellular biological process, protein binding and polymer complex. Using RNA sequencing technology, Zhang et al. (2017) studied the skin tissue of Superfine merino wool sheep (SM) and Small tail han sheep (STH) and coarse wool sheep. He obtained 435 differentially expressed genes, including 127 downregulated genes and 308 up-regulated genes. GO and KEGG enrichment analysis showed that the genes, highly expressed in SM, were mainly related to hair follicle stem cell markers. However, wool fiber composition and lipid metabolism pathway, and genes related to wool fiber structure are mainly concentrated in KRT and KRTAP gene family. On the other hand, the genes highly expressed in STH were mainly related to skin keratinization and muscle composition. Similarly, the research related to miRNAs expression profile in the skin and hair follicles of Liaoning cashmere goat and Aohan fine wool sheep during the stationary period were also surveyed by solexa deep sequencing technology (Li et al. 2016). A total of 1910 known miRNAs and 2261 new miRNAs were identified. Among them, 107 newly discovered miRNAs and 1246 known miRNAs were differentially expressed in the two breeds. Go and KEGG enrichment analyses showed that these miRNAs played an important role in hair follicle growth and development. As an important factor in animal breeding, coat color is produced by skin melanocytes and determined by the amount and type of melanin released (Ito et al. 2000; Ito and Wakamatsu 2008). In addition, the skin tissues of three white and black wool Sunit sheep were collected and used for transcriptome sequencing, and found 845 new differentially expressed genes (Fan $e t$ al. 2013). Of which 107 and 738 genes were highly expressed in black sheep and white sheep skin, respectively. And 49 known hair color related genes were expressed in skin tissues, 13 genes were highly expressed in black sheep skin, mainly in DCT, MATP, TYR and TYRP1 which were related to melanin and its receptor. Some important genes function was shown in the Table 1.

\section{Research progress of non-coding RNA in hair follicle development}

In the process of hair follicle development and growth cycle, it is regulated by many factors and pathways. Through the influence of these regulatory mechanisms, hair follicles cycle in the process of growth, regression and rest. As an important regulator, IncRNA plays a significant role in hair follicle development. Long noncoding RNA (LncRNA) is a kind of eukaryotic transcripts with a length of more than $200 \mathrm{nt}$ and nocoding capacity. It is the key regulator of gene coding proteins. It regulates gene expression at both transcriptional and post transcriptional levels. It is widely distributed in animal and plant genomes. As an important regulator, IncRNA participates in life cycle, cell differentiation and some disease-related biological processes. At present, some IncRNAs and miRNAs related to skin biology have been reported. A large number of studies have shown that lncRNA plays an important role in the process of skin hair follicle development, participates in regulating the expression of hair follicle and hair fiber growth related genes. It is closely related to hair follicle development and periodic changes, and can play its biological functions by interacting with many growth factors and signal pathways. Many studies found that 6127 lncRNAs were expressed during the growth and resting stages of cashmere, among which 54 were significantly different (32 up-regulated and 22 down regulated). And targeted knockout of the differentially expressed lncRNA-5479 showed that it was closely related to the growth period of hair follicles and participated in the formation of keratin (Guo 2015; Feng 2016). Moreover, IncRNAs and miRNAs usually work together to occur in primary hair follicle induction of sheep fetal skin (Nie et al. 2018). It was also found that 36 of the differentially expressed lncRNAs were up-regulated and 26 were downregulated. The down-regulated IncRNAs interacted with keratin (krt14 and krt15), BMP signal (sostdc1), Wnt signal (wnt16 and SFRP1) and laminin (LAMA1), which mainly affected the development of epidermis and wool substrate. Additionally, the transcription patterns between resting and growing stages of cashmere goat secondary hair follicles were definituded and found 13 differentially expressed IncRNAs, of which 6 were up-regulated in growth period, and 4 were up-regulated in resting period (Bai et al. 2018). The results showed that the expression levels of IncRNA000133 (Zheng et al. 2019), IncRNA-H19 (Zhu et al. 2018) and IncRNA-HOTAIR (Jiao et al. 2019) in cashmere goat hair follicle development and growth stage were significantly higher than those in resting period. Combined with promoter methylation analysis, the hypermethylation of IncRNA-H19, IncRNA HOTAIR and IncRNA-000133 may be involved in the expression inhibition of Cashmere goat secondary hair follicles. In addition, many studies predicted and identified the target genes of differential lncRNAs in the induction period of secondary hair follicle of fine wool sheep (Liu 2016; Wang et al. 2018). Where, it was found that lncRNA may also participate in the regulation of fine wool follicle morphology through nod like receptor signaling pathway, leishmaniasis and NF kappaB signaling pathway occurrence and development cycle.

\section{Research progress on GWAS of sheep wool traits}

GWAS refers to the search for single nucleotide variations related to the main economic traits of animals and plants 
Hair Follicle Development and Wool Production Traits / Intl J Agric Biol, Vol 25, No 2, 2021

Table 1: List of some important gene functions

\begin{tabular}{|c|c|c|c|c|}
\hline Gene name & Function & Experimental technology & Species & Reference \\
\hline FAM101A, COL21A1 and FACIT & Dirty wool & SNP50 bead chip & sheep & $\begin{array}{l}\text { Ebrahimi et al. } \\
\text { (2017) }\end{array}$ \\
\hline MAP2 & Prenatal hair follicle density & GWAS & pig & $\begin{array}{l}\text { Jiang et al. } \\
\text { (2019) }\end{array}$ \\
\hline PML、LAMC2 and PDGFD & Wool fiber bending properties & OvineSNP50 BeadChip & Chinese Merino sheep & Di et al. (2015) \\
\hline FGF5 & Length and yield of wool & CRISPR/cas9 and RT-PCR & sheep & Liu et al. (2011) \\
\hline KIF16B & Coefficient of variation of wool fiber diameter & GWAS & Chinese Merino sheep & $\begin{array}{l}\text { Wang et al. } \\
\text { (2014) }\end{array}$ \\
\hline WNT10b & $\begin{array}{l}\text { hair stem and inner promotes differentiation of } \\
\text { skin epithelial cells. }\end{array}$ & Cell validation experiment & mouse & $\begin{array}{l}\text { Mikkola and } \\
\text { Millar (2006) }\end{array}$ \\
\hline
\end{tabular}

within the biological genome. Wang et al. (2014) used GWAS analysis on the wool yield and wool quality of 765 Chinese Merino sheep and found 28 SNPs sites significantly related to wool fiber diameter, its change rate and fineness and crimp. Gene annotation showed that $43 \%$ of SNPs were involved in the biological process of hair growth and development. Moreover, some SNPs were found significantly associated with wool staple crimp frequency, wool length and wool yield in Chinese Merino sheep. Further, it was found that five SNPs related significantly to wool staple crimp frequency which were located on chromosomes 2,12 , 15 and 18, respectively (Di et al. 2015). Two SNPs which were significantly related to wool length could be located on chromosomes 2 and 15, and 3 SNPs related significantly to wool yield could be located to chromosomes 6,7 and 1 . Gene annotation and analysis showed that SNPs significantly related to wool staple crimp frequency were located in IKZF2, LAMC2, ARHGAP42, GABRG3 and PML. And SNPs significantly related to wool length and wool yield were located or adjacent to FIBIN, HSD17b11 and PIAS1, which involved in wool growth and development related biological processes. In addition, the amount of dirty wool of 96 Baluchi sheep was used for GWAS analysis by ovine SNP50 bead chip (Ebrahimi et al. 2017), and found that three SNPs significantly related at chromosome level were located on chromosome 17 and 20 respectively. Gene annotation revealed that these three loci were located or adjacent to FAM101A, COL21A1 and FACIT.

\section{Conclusion and outlook}

Hair follicle development and wool trait have received greater attention in ruminats, especially sheep. At present, the research on transcriptome, IncRNA and GWAS in hair follicle development and wool trait mainly focuses on the screening and identification of candidate genes, IncRNA and genetic variation sites (including SNP and CNV). However, the mechanism of these candidate gene or regulatory molecule is still unclear. With the rapid development of molecular technology and gene editing technology, it is necessary to reveal the molecular mechanism of hair follicle periodic growth more deeply and accurately. It also provides new research direction and ideas for livestock breeding and hair follicle development and wool trait improvement.

\section{Author Contributions}

Wu Sun wrote the first draft, Shike Ma designed the experiment and Yuhong Ma revised the text.

\section{References}

Bai WL, SJ Zhao, ZY Wang, YB Zhu, YL Dang, YY Cong, HL Xue, W Wang, L Deng, D Guo (2018). LncRNAs in Secondary Hair Follicle of Cashmere Goat: Identification, Expression, and Their Regulatory Network in Wnt Signaling Pathway. Anim Biotechnol 29:199-211

Bidinost F, DL Roldan, AM Dodero, EM Cano, HR Taddeo, JP Mueller, MA Poli (2008). Wool quantitative trait loci in Merino sheep. Small Rumin Res 74:113-118

Blessing M, LB Nanney, LE King, CM Jones, BLM Hogan (1993). Transgenic mice as a model to study the role of TGF-beta-related molecules in hair follicles. Genes Dev 7:204-215

Bond JJ, PC Wynn, GPM Moore (1996). Effects of epidermal growth factor and transforming growth factor alpha on the function of wool follicles in culture. Arch Dermatol Res 288:373-382

Bongiorni S, G Chillemi, G Prosperini, S Bueno, A Valentini, L Pariset (2009). A tool for sheep product quality: Custom microarrays from public databases. Nutrients 1:235-250

Botchkarev VA, NV Botchkareva, AA Sharov, K Funa, O Huber, BA Gilchrest (2002). Modulation of BMP signaling by noggin is required for induction of the secondary (nontylotrich) hair follicles. $J$ Invest Dermatol 118:3-10

Botchkarev VA, NV Botchkareva, W Roth, M Nakamura, L Chen, W Herzog, G Lindner, JA Mcmahon, C Peters, R Lauster (1999). Noggin is a mesenchymally derived stimulator of hair-follicle induction. Nat Cell Biol 1:158-164

Cano EM, G Marrube, DL Roldan, F Bidinost, M Abad, D Allain, D Vaiman, HR Taddeo, MA Poli (2007). QTL affecting fleece traits in Angora goats. Small Rumin Res 71:158-164

Chen W, XB Fu, TZ Sun, Z Zhao, Y Yang, Z Sheng (2002). The expression characteristics and their biological significance of epidermal growth factor and its receptor in fetal and postnatal skins. Mod Rehabil 6:1128-1130

Chong C, P Wu, F Zhang, X Xu, M Yu, RB Widelitz, T Jiang, L Hou (2003). Adaptation to the sky: Defining the feather with integument fossils from mesozoic China and experimental evidence from molecular laboratories. J Exp Zool 298:42-56

Cros DLD, K Isaacs, GPM Moore (1992). Localization of epidermal growth factor immunoreactivity in sheep skin during wool follicle development. J Invest Dermatol 98:109-115

Di J, J Liu, X Xu, A Lazart, L Yu (2015). Genome-wide association studies on the wool staple crimp frequency in Chinese Merino sheep (Xinjiang type). Xinjiang Agric Sci 52:2129-2135

Ebrahimi F, M Gholizadeh, G Rahimimianji, A Farhadi (2017). Detection of QTL for greasy fleece weight in sheep using a $50 \mathrm{~K}$ single nucleotide polymorphism chip. Trop Anim Health Product 49:1657-1662

Fan R, J Xie, J Bai, H Wang, X Tian, R Bai, X Jia, L Yang, Y Song, M Herrid, W Gao, XHe, J Yao, GW Smith, CDong (2013). Skin transcriptome profiles associated with coat color in sheep. BMC Genomics 14; Article 389 
Feng S (2016). Preliminary study on the epigenetics of wool sac development based on methylation and IncRNA. Master degree. Yangling: Northwest A\&F University

Fuchs E, S Raghavan (2002). Getting under the skin of epidermal morphogenesis. Nat Rev Genet 3:199-209

Galbraith H (2010). Fundamental hair follicle biology and fine fibre production in animals. Animal 4:1490-1509

Guo Y (2015). Screening of Specific IncRNAs for Cashmere Periodic Growth. Master degree. Yangling: Northwest A\&F University. 2015

Hao L, Y Li, W Jiang, X Wang, D Shi (2006). Morphological observation of rat embryonic hair follicle development. J Northeast Norm Univ-Nat Sci 38:114-116

Hirokawa N, Y Tanaka (2015). Kinesin superfamily proteins (KIFs): Various functions and their relevance for important phenomena in life and diseases. Exp Cell Res 334:16-25

Hu R, ZY Fan, BY Wang, SL Deng, XS Zhang, JL Zhang, HB Han, ZX Lian (2017). RAPID COMMUNICATION: Generation of FGF5 knockout sheep via the CRISPR/Cas9 system. J Anim Sci 95:2019-2024

Ito S, K Wakamatsu (2008). Chemistry of Mixed Melanogenesis-Pivotal Roles of Dopaquinone. Photochem Photobiol 84:582-592

Ito S, K Wakamatsu, H Ozeki (2000). Chemical analysis of melanins and its application to the study of the regulation of melanogenesis. Pigment Cell Res 13:103-109

Jamora C, R Dasgupta, P Kocieniewski, E Fuchs (2003). Links between signal transduction, transcription and adhesion in epithelial bud development. Nature 422:317-322

Janich P, G Pascual, A Merlossuarez, E Batlle, JA Ripperger, U Albrecht, HM Cheng, K Obrietan, LD Croce, SA Benitah (2011). The circadian molecular clock creates epidermal stem cell heterogeneity. Nature 480:209-214

Jiang Y, Y Jiang, H Zhang, M Mei, H Song, X Ma, L Jiang, Z Yu, Q Zhang, $X$ Ding (2019). A mutation in MAP2 is associated with prenatal hair follicle density. FASEB J 33:14479-14490

Jiao Q, RH Yin, SJ Zhao, ZY Wang, YB Zhu, W Wang, YY Zheng, XB Yin, DY Guo, SQ Wang, YX Zhu, WL Bai (2019). Identification and molecular analysis of a IncRNA-HOTAIR transcript from secondary hair follicle of cashmere goat reveal integrated regulatory network with the expression regulated potentially by its promoter methylation. Gene 688:182-192

Kopan R, H Weintraub (1993). Mouse notch: Expression in hair follicles correlates with cell fate determination. J Cell Biol 121:631-641

Li J, H Qu, H Jiang, Z Zhao, Q Zhang (2016). Transcriptome-wide comparative analysis of microRNA profiles in the telogen skins of Liaoning Cashmere goats (Capra hircus) and fine-wool sheep (Ovis aries) by Solexa deep sequencing. DNA Cell Biol 35:696-705

Lin CM, TX Jiang, RB Widelitz, CM Chuong (2006). Molecular signalling in feather morphogenesis. Curr Opin Cell Biol 18:730-741

Liu N, CL Wang, JN He, M Cheng, KD Liu, JF Liu, JS Zhao (2015). The development and morphological structure of skin hair follicles in different parts of Aohan fine-wool sheep. Chin J Anim Husb 17:7-11

Liu N, H Li, K Liu, J Yu, R Bu, M Cheng, W De, J Liu, G He, J Zhao (2014). Identification of skin-expressed genes possibly associated with wool growth regulation of Aohan fine wool sheep. BMC Genet 15; Article 144

Liu S (2016) Identification and Functional Analysis Of Differentially Expressed LncRNA Target Genes During the Induction Period of Fine-wool Sheep Secondary Hair Follicle Morphogenesis. Master degree. Gansu Agricultural University, Lanzhou, China

Liu W, B Jia, G Shi, J Ren, K Liu, R Ma (2011). Cloning, expression and RNA interference of sheep FGF5 gene. Genetic 33:982-988

Mikkola ML, SE Millar (2006). The Mammary Bud as a Skin Appendage: Unique and Shared Aspects of Development. J Mammary Gland Biol 11:187-203

Nanney LB, M Magid, CM Stoscheck, LE King (1984). Comparison of epidermal growth factor binding and receptor distribution in normal human epidermis and epidermal appendages. J Invest Dermatol 83:385-393
Nie Y, S Li, X Zheng, W Chen, X Li, Z Liu, Y Hu, H Qiao, Q Qi, Q Pei (2018). Transcriptome reveals long non-coding RNAs and mRNAs involved in primary wool follicle induction in carpet sheep fetal skin. Front Physiol 9; Article 446

Norris BJ, NI Bower, WJ Smith, GR Cam, A Reverter (2005). Gene expression profiling of ovine skin and wool follicle development using a combined ovine-bovine skin cDNA microarray. Aust J Exp Agric 45:867-877

Ollier S, C Robertgranie, L Bernard, Y Chilliard, C Leroux (2007). Mammary Transcriptome analysis of food-deprived lactating goats highlights genes involved in milk secretion and programmed cell death. J Nutr 137:560-567

Ota Y, Y Saitoh, S Suzuki, K Ozawa, M Kawano, T Imamura (2002). Fibroblast growth factor 5 inhibits hair growth by blocking dermal papilla cell activation. Biochem Bioph Res Commun 290:169-176

Packer AI, D Janewit, L Mclean, AA Panteleyev, AM Christiano, DJ Wolgemuth (2000). Hoxa4 expression in developing mouse hair follicles and skin. Mech Dev 99:153-157

Paus R, G Cotsarelis (1999). The biology of hair follicles. New Engl J Med 341:491-497

Penagaricano F, P Zorrilla, H Naya, C Robello, JI Urioste (2012). Gene expression analysis identifies new candidate genes associated with the development of black skin spots in Corriedale sheep. J Appl Genet 53:99-106

Purvis IW, IR Franklin (2005). Major genes and QTL influencing wool production and quality: A review. Genet Select Evol 37:1-11

Rhee H, L Polak, E Fuchs (2006). Lhx2 Maintains Stem Cell Character in Hair Follicles. Science 312:1946-1949

Schmidtullrich R, R Paus (2005). Molecular principles of hair follicle induction and morphogenesis. Bioessays 27:247-261

Schneider MR, R Schmidtullrich, R Paus (2009). The hair follicle as a dynamic miniorgan. Curr Biol 19:132-142

Shang L, ND Pruett, A Awgulewitsch (2002). Hoxc12 expression pattern in developing and cycling murine hair follicles. Mech Dev 113:207-210

Smith WJ, Y Li, A Ingham, E Collis, SM Mcwilliam, TJ Dixon, BJ Norris, SI Mortimer, RJ Moore, A Reverter (2010). A genomics-informed, SNP association study reveals FBLN1 and FABP4 as contributing to resistance to fleece rot in Australian Merino sheep. BMC Vet Res 6; Article 27

Veen CVD, B Handjiski, R Paus, S Mullerrover, M Maurer, SB Eichmuller, G Ling, U Hofmann, K Foitzik, L Mecklenburg (1999). A comprehensive guide for the recognition and classification of distinct stages of hair follicle morphogenesis. J Invest Dermatol 113:523-532

Wang J, T Wang, D Wang, Y Zhang, X Wang (2018). Research progress in the regulation of animal hair follicle development by miRNA and lncRNA. Chin J Anim Husb 54:35-40

Wang Z, H Zhang, H Yang, S Wang, E Rong, W Pei, H Li, N Wang (2014). Genome-wide association study for wool production traits in a Chinese Merino sheep population. PLoS One 9; Article e107101

Zhang L, F Sun, H Jin, BP Dalrymple, Y Cao, T Wei, T Vuocolo, M Zhang, Q Piao, A Ingham (2017). A comparison of transcriptomic patterns measured in the skin of Chinese fine and coarse wool sheep breeds. Sci Rep 7; Article 14301

Zhang Y, J Yin, J Li, C Li (2007). Study on the structure and morphogenesis of wool sacs of the wool goats of Albas, Inner Mongolia. $J$ Integr Agric 5:1017-1023

Zhang Z, S Wang, H Wang, J Zhao (2009). Study on differentially expressed genes in jining grey goats. J Shandong Univ-Nat Sci 44:7-10

Zheng Y, Z Wang, Y Zhu, W Wang, M Bai, Q Jiao, Y Wang, S Zhao, X Yin, D Guo (2019). LncRNA-000133 from secondary hair follicle of Cashmere goat: Identification, regulatory network and its effects on inductive property of dermal papilla cells. Anim Biotechnol 31:122-134

Zhu YB, ZY Wang, RH Yin, Q Jiao, SJ Zhao, YY Cong, HL Xue, D Guo, SQ Wang, YX Zhu (2018). A lncRNA-H19 transcript from secondary hair follicle of Liaoning cashmere goat: Identification, regulatory network and expression regulated potentially by its promoter methylation. Gene 641:78-85 